\title{
Expression and clinical value of CD105 in renal cell carcinoma based on data mining in The Cancer Genome Atlas
}

\author{
DONGHUI SHI ${ }^{1,2}$, JIANPING CHE ${ }^{1}$, YANG YAN ${ }^{1}$, BO PENG $^{1}$, XUDONG YAO $^{1}$ and CHANGCHENG GUO ${ }^{1}$ \\ ${ }^{1}$ Department of Urology, Shanghai 10th People's Hospital, Tongji University, Shanghai 200072; \\ ${ }^{2}$ Department of Urology, Suzhou Wu Zhong People's Hospital, Suzhou, Jiangsu 215128, P.R. China
}

Received July 16, 2018; Accepted November 30, 2018

DOI: $10.3892 /$ etm.2019.7493

\begin{abstract}
The objective of the present study was to assess the expression of CD105 and its association with overall survival in three subtypes of renal cell carcinoma (RCC), namely clear cell (cc)RCC, papillary (p)RCC and chromophobe (ch)RCC. Data regarding the transcriptome and copy number of genes in RCC tumor samples and survival were obtained from The Cancer Genome Atlas. Bioinformatics analysis revealed that CD105 is overexpressed in ccRCC tumor tissue vs. normal renal tissue, and a higher CD105 copy number in ccRCC tissues was significantly associated with longer patient survival. The effect of the mRNA expression of CD105 in all three types of RCC and the copy number in pRCC and chRCC on patient survival was insignificant, but certain trends were observed. In addition, CD105 mRNA expression was associated with the metastasis and tumor stage, as well as pathological stage in ccRCC and pRCC. Pathway enrichment analysis revealed that CD105 may, through translation initiation of associated genes, promote RCC progression. The results of the present study suggest that in RCC tumors, the association of CD105 with different stages is complex. To evaluate the role of CD105 in RCC, its function should be assessed in addition to its expression. The exact influence of CD105 mRNA expression and copy number in RCC tumors on patient survival and the underlying mechanisms require further elucidation.
\end{abstract}

\section{Introduction}

Renal cell carcinoma (RCC) is the most common type of malignancy of the kidney, accounts for 2-3\% of all malignancies in adults (1) and its incidence and mortality are currently

Correspondence to: Professor Changcheng Guo or Professor Xudong Yao, Department of Urology, Shanghai 10th People's Hospital, Tongji University, 301 Yanchang Road, Shanghai 200072, P.R. China

E-mail: greatwall063030@126.com

E-mail: yaoxudong67@sina.com

Key words: renal cell carcinoma, CD105, The Cancer Genome Atlas, survival, clinical value on the rise (2). In the USA, RCC is the 6th leading cause of cancer-associated deaths in men and the 8th leading cause in women (3). At present, surgery is the standard treatment for primary RCC, while seven targeted therapies have been approved for metastatic RCC by the Food and Drug Association (4). However, the treatment of RCC remains a huge challenge due to the generally poor response to chemotherapy and radiotherapy (5-7). Despite persistent research efforts in the past several decades, only little progress has been made regarding the early diagnosis and treatment of RCC (8). To reach this goal, it is necessary to identify early diagnostic biomarkers and therapeutic targets using novel approaches, such as a bioinformatics search.

Cancer stem cells (CSCs) were first identified in 3 types of solid tumor in the early 2000s (9). Now, CSCs have been identified in various cancer types, including RCC (10). Targeting of CSCs has become an important strategy to treat cancer. RCC exhibits a poor response to chemotherapy and radiotherapy due to the survival of CSCs (5-7), and it is important to identify molecular markers to isolate and characterize the CSCs among the tumor cells; of note, targeting of CSCs in RCC has provided a novel treatment strategy, particularly for metastatic RCC (11).

Recently, CD105 has been described as a novel RCC CSC marker (12). Therefore, the present study aimed to assess whether tumoral CD105 has a prognostic value in RCC. CD105 (endoglin) is the receptor for transforming growth factor (TGF). CD105 regulates TGF- $\beta$ signaling by interacting with TGF- $\beta$ receptors I and/or II. Several studies have indicated that CD105 contributes to the development of blood vessels and angiogenesis, and is essential for tumor growth and the development of metastasis. In addition, CD105 is a prominent marker for mesenchymal stem cells (MSCs) (13-15). In RCC, CD105 has been reported as a potential prognostic marker and CSC marker. Bussolati et al (16), Hasmim et al (17) and Dallas et al (15) indicated that $\mathrm{CD} 105^{+} \mathrm{RCC}$ cells had stronger features of CSCs compared with $\mathrm{CD} 105^{-}$cells. Furthermore, these $\mathrm{CD} 105^{+}$cells expressed MSC markers including CD44, CD146 and CD73, embryonic stem cell markers including Nanog and Oct4, and embryonic renal marker paired box-2, but lacked differentiated epithelial markers. Recently, Saeednejad Zanjani et al (13) performed an analysis of 186 clear cell (cc)RCC samples, revealing that CD105 expression was associated with more aggressive tumor behavior, more advanced disease and worse prognosis. However, in other 
types of RCC, including papillary (p)RCC, it was not possible to confirm CD105 as a CSC marker (12). Further studies questioned the use of CD105 as a renal CSC marker, as CD105- cells also exhibited CSC-like features $(10,18)$. These inconclusive and conflicting results suggest that CD105 in different types of $\mathrm{RCC}$ requires further study.

The Cancer Genome Atlas (TCGA) database includes gene expression data obtained by RNA-sequencing (seq) for cohorts of $604 \mathrm{ccRCC}, 320 \mathrm{pRCC}$ and 89 chromophobe (ch)RCC cases with the clinical outcome data available. In the present study, it was attempted to assess the expression and function of CD105 in RCC based on mining of TCGA data. The possible functional role of CD105 in RCC may have clinical implications. If CD105 has a function in RCC, strategies to target CD105 in RCC may represent a novel therapeutic strategy.

\section{Materials and methods}

Data retrieval from TCGA. Gene expression data obtained by RNA-seq for cohorts of 604 ccRCC, 320 pRCC and 89 chRCC cases that have clinical outcome data available were extracted from TCGA (http://xena.ucsc.edu/). These datasets included $\sim 20,500$ data-points each for ccRCC, pRCC and chRCC. Clinical information for each patient, including survival status, time to last follow-up and gender, was also extracted from TCGA. CD105 mRNA and protein expression data and matching clinical information were also retrieved from TCGA for these patients. In addition, data on the CD105 copy number and matching clinical information were retrieved from TCGA for $526 \mathrm{ccRCC}$ patients.

Survival analysis. Patients were stratified into two groups (high and low) based on the mean levels of CD105 mRNA expression or copy number. Kaplan-Meier analysis was performed using GraphPad prism (version 7; GraphPad Software Inc., La Jolla, CA, USA) or Xena (http://xena.ucsc.edu/).

CD105-associated gene expression and enriched pathway analysis. The whole gene expression (RNA-seq) data of the 10 patients with the highest CD105 expression and 10 patients with lowest CD105 expression was obtained from TCGA. The differentially expressed genes of these two groups were analysis by webMeV (version 1.0; http://mev.tm4.org/\#/datasets/tcga). Then the pathways enriched by the upregulated and downregulated genes associated with CD105 from the TCGA dataset were analysis by webMeV and the dataset was calculated using the voom function.

Statistical analysis. All statistical analyses were carried out with SPSS 19.0 (IBM Corp., Armonk, NY, USA). Differences in mean values between two groups were analyzed by a two-tailed Student's t-test and the mean values of $>2$ groups were compared with one-way analysis of variance. Multiple comparison between the groups was performed using Student-Newman-Keuls method. $\mathrm{P} \leq 0.05$ was considered statistically significant.

\section{Results}

CD105 expression in tumor and normal tissue in different cancer types. To confirm the expression of CD105, the expression of
CD105 in tumor and normal tissue from the TCGA database was analyzed in different types of cancer using firebrowse software (http://firebrowse.org/\#). The results indicated that the expression of CD105 is different in different cancer types (Fig. 1A). The expression of CD105 in tumor tissue is higher compared normal tissue in patients with thyroid carcinoma, while in patients with liver hepatocellular carcinoma, the expression of CD105 was higher in normal tissue compared with tumor tissue. Furthermore, it was identified that the expression of CD105 in ccRCC tumor tissue was significantly higher compared with that of normal renal tissue $(\mathrm{P}=0.03)$. However, for $\mathrm{pRCC}$ and chRCC, the expression of CD105 in normal tissue was significantly higher compared with that in the tumor tissue $(\mathrm{P}=0.04$ and $\mathrm{P}=0.01$; Fig. 1B). These results suggest that CD105 may play an important role in ccRCC, but not in pRCC and chRCC.

Prognostic value of CD105 expression in RCC. CD105 mRNA expression levels and clinical follow-up data of 604 cases of ccRCC, the most common subtype of RCC, were obtained from TCGA using Xena. The clinicopathological information of the patients are listed in Table I. Cases were assigned to CD105-high or CD105-low groups using the median CD105 mRNA expression as a cutoff. Time to death was plotted in a Kaplan-Meier curve for those cases exhibiting expression of CD105 transcripts above the median $(n=304)$ and equal to or below the median $(n=301)$. The results indicated no significant difference between the two groups $(\mathrm{P}=0.25)$, although the curves exhibited a trend, with those cases with a higher expression of CD105 surviving for longer (Fig. 2A).

Similar analyses were performed for pRCC $(n=318)$ and chRCC patients $(n=87)$. Among patients with pRCC, there was also no significant difference in survival between the CD105 high and low expression groups $(\mathrm{P}=0.44)$. Similar results were also obtained for chRCC with a P-value of 0.54 . These results suggest that, although there was a trend of the CD105 high expression group surviving for longer in the three types of RCC, CD105 expression had no significant influence on survival.

The prognostic value of gender in RCC was also assessed, as it was reported that $\mathrm{RCC}$ has a gender bias in incidence with a male-to-female ratio of 2.3:1 (19). In the current study, the male-to-female ratio was depicted in Table I. However, no significant impact of gender on survival was identified in ccRCC, pRCC and chRCC (data not shown). These results suggest that while the incidence of RCC is higher in males, the outcome of RCC in males and females is similar.

$C D 105$ expression in different stages and grades of $R C C$. The present study also assessed the association of CD105 expression with different tumor (T) and metastasis (M) stages, as well as the pathological stage, in the three types of RCC. The results indicated that the expression of CD105 exhibited no significant difference between M1 and M0 $(\mathrm{P}=0.90)$. Similarly, no significance was obtained regarding the differences between different $\mathrm{T}$-stages (T1 vs. T2, P=0.24). However, CD105 was significantly higher expressed in stage II than in stage I tumors $(\mathrm{P}=0.04)$, while CD105 expression in stage IV tumors was lower than that in stage II tumors, but with no statistical significance $(\mathrm{P}=0.18)$. The same result was obtained for $\mathrm{pRCC}$ (Fig. 3B), where CD105 was higher expressed in stage II than in stage I tumors $(\mathrm{P}=0.04)$. Furthermore, in pRCC, CD105 was 
A CD105 differential plot

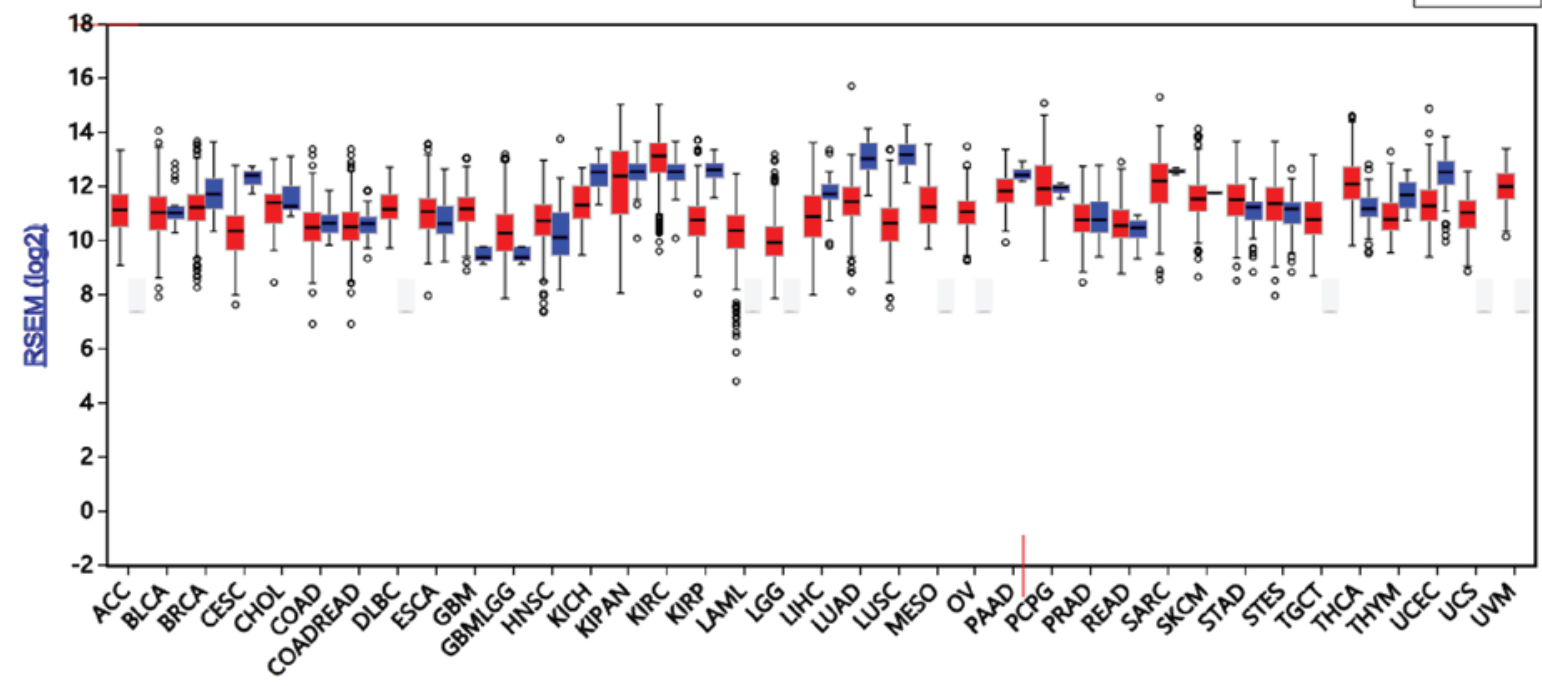

B
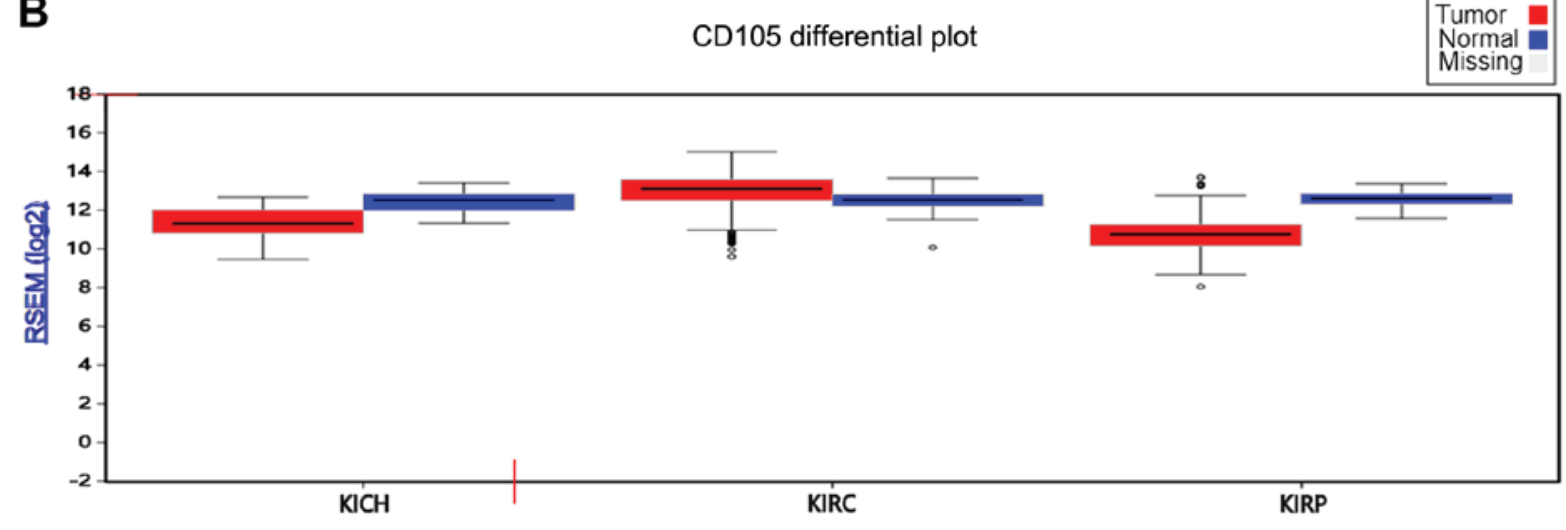

Figure 1. CD105 expression in normal and tumor tissues in different cancer types. (A) CD105 expression in different cancer and normal tissues. (B) CD105 expression in three renal cell carcinoma and normal kidney tissues. The top, middle and bottom lines of the boxes indicate the third quartiles, median and first quartiles, respectively. The whiskers indicate the standard deviations and the circles indicate the values beyond the standard deviations. The different cancer types are displayed on the $\mathrm{x}$-axis. ACC, adrenocortical carcinoma; BLCA, bladder urothelial carcinoma; BRCA, breast invasive carcinoma; CESC, cervical squamous cell carcinoma and endocervical adenocarcinoma; CHOL, cholangiocarcinoma; COAD, colon adenocarcinoma; COADREAD, colorectal cancer; DLBC, Lymphoid Neoplasm Diffuse Large B-cell Lymphoma; ESCA, esophageal carcinoma; GBM, glioblastoma multiforme; GBMLGG, glioma; HNSC, head and neck squamous cell carcinoma; KICH, kidney chromophobe; KIPAN, pan-kidney cohort (KICH+KIRC+KIRP); KIRC, kidney renal clear cell carcinoma; KIRP, kidney renal papillary cell carcinoma; LAML, acute myeloid leukemia; LGG, brain lower grade glioma; LIHC, liver hepatocellular carcinoma; LUAD, lung adenocarcinoma; LUSC, lung squamous cell carcinoma; MESO, mesothelioma; OV, ovarian serous cystadenocarcinoma; PAAD, pancreatic adenocarcinoma; PCPG, pheochromocytoma and paraganglioma; PRAD, prostate adenocarcinoma; READ, rectum adenocarcinoma; SARC, sarcoma; SKCM, skin cutaneous melanoma; STAD, stomach adenocarcinoma; STES, stomach and esophageal carcinoma; TGCT, testicular germ cell tumors; THCA, thyroid carcinoma; THYM, thymoma; UCEC, uterine corpus endometrial carcinoma; UCS, uterine carcinosarcoma; UVM, uveal melanoma; RSEM, RNA-seq by expectation-maximization.

Table I. Clinicopathological information.

\begin{tabular}{lccc}
\hline Variable & ccRCC & pRCC & chRCC \\
\hline Total number of patients & 604 & 320 & 89 \\
Female & 210 & 79 & 41 \\
Male & 394 & 241 & 48 \\
Sex ratio (male:female) & 1.88 & 2.81 & 1.22 \\
Age (mean \pm standard deviation) & $61 \pm 0.48$ & $62 \pm 0.43$ & $52 \pm 0.39$ \\
\hline
\end{tabular}

higher expressed in T3 than in T2 tumors $(\mathrm{P}=0.02)$. However, the expression of CD105 exhibited no difference between different T, M and pathological stages in chRCC (Fig. 3C).
These results suggest that the function of CD105 in RCC at different stages is complex.

Gene copy number of CD105 in RCC. A possible mechanism for the high expression of CD105 in RCC is the gene copy number. Therefore, the copy number of CD105 in RCC samples was analysed in the present study. Data on CD105 copy number, expression levels and clinical follow-up of 526 cases of ccRCC were obtained from TCGA using Xena (http://xena.ucsc. edu/). Cases were assigned into CD105-high or CD105-low copy number groups using the median CD105 copy number as the cutoff. Time to death was plotted in a Kaplan-Meier curve for those cases with a CD105 copy number above the median $(n=265)$ and equal to or below the median $(n=267)$. A significant difference in survival was identified between 

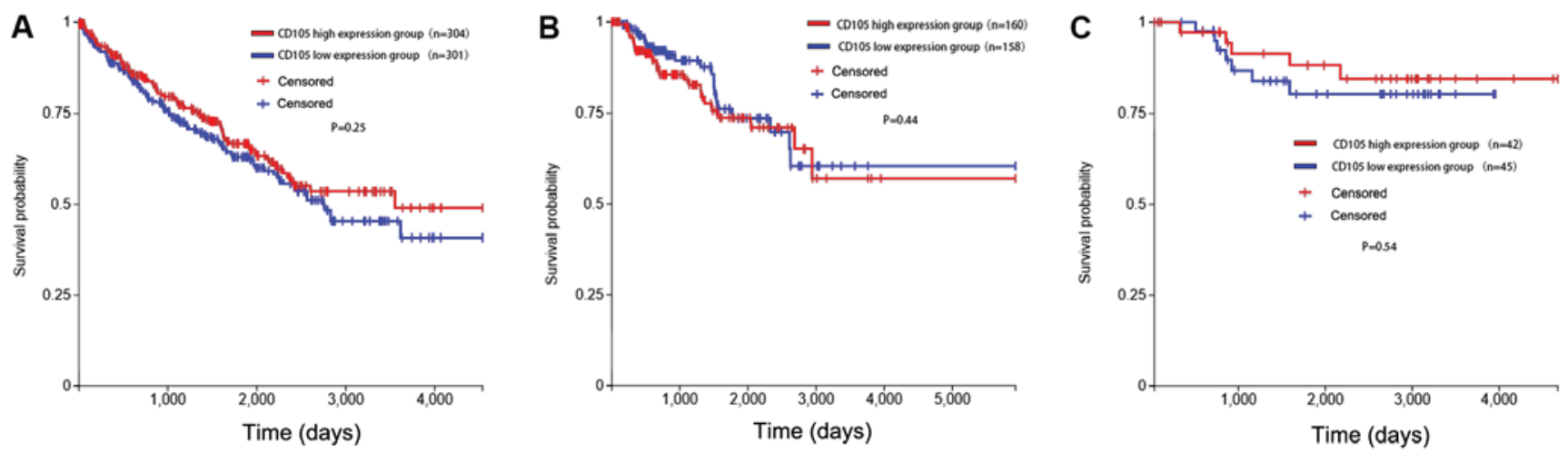

Figure 2. mRNA expression of CD105 and its association with survival in ccRCC, pRCC and chRCC. Kaplan-Meier analysis for the influence of CD105 mRNA expression on overall survival in (A) ccRCC, (B) pRCC and (C) chRCC. RCC, renal cell carcinoma; ccRCC, clear cell RCC; pRCC, papillary RCC; chRCC, chromophobe RCC.

A

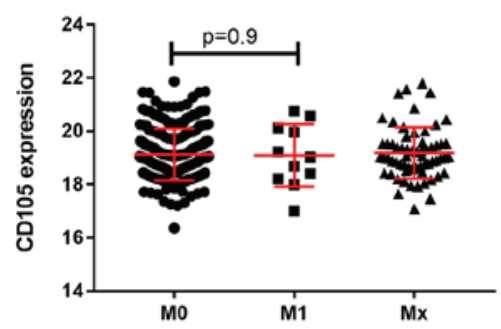

B

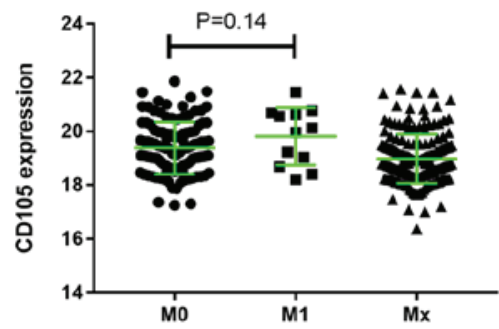

C

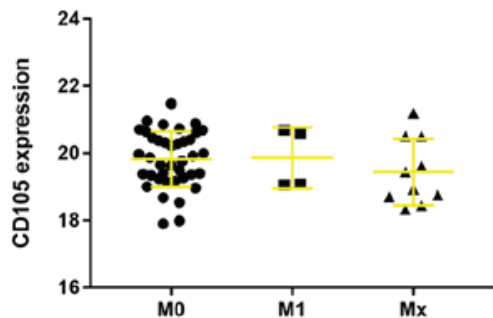

$\operatorname{ccRCC}$

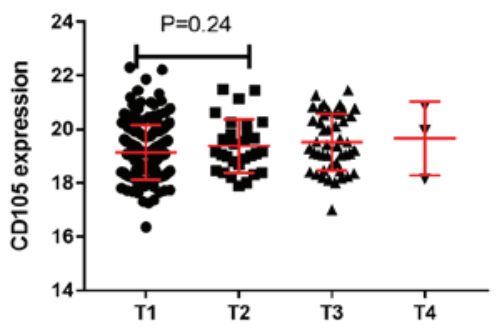

pRCC
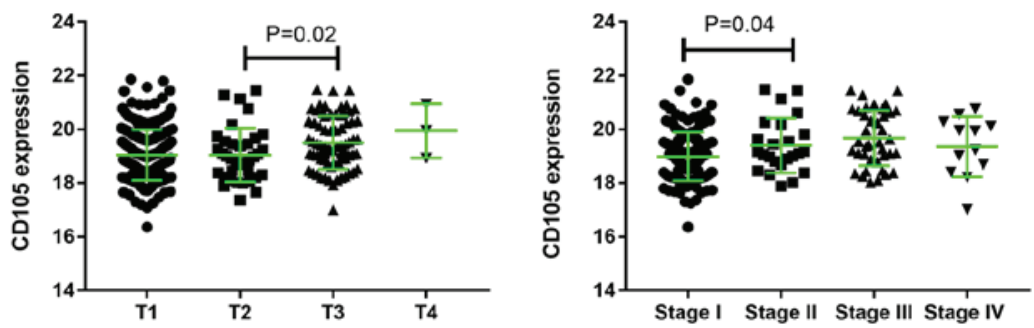

$\operatorname{chRCC}$

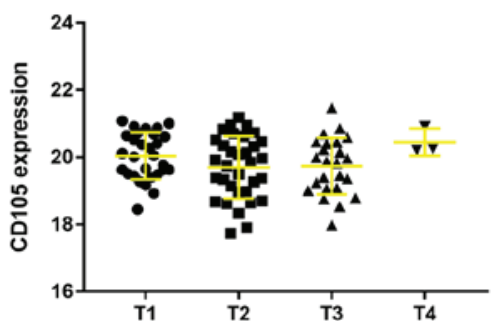

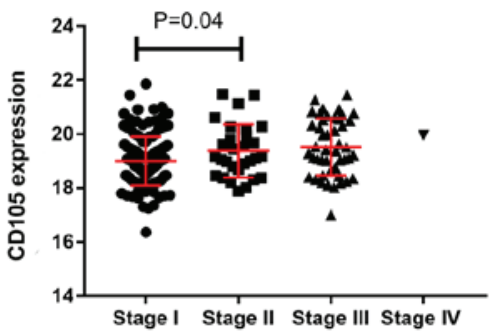

Stage I Stage II Stage III Stage IV

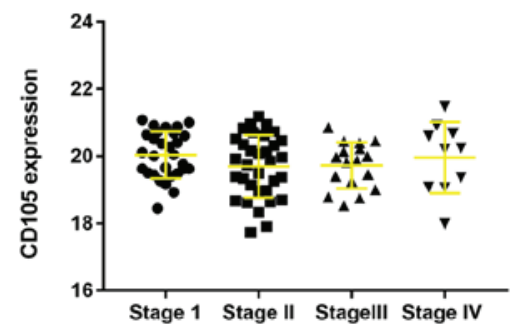

Figure 3. mRNA expression of CD105 and its association with $\mathrm{M}$ and $\mathrm{T}$ stage, as well as pathological stage, in ccRCC, pRCC and chRCC. (A-C) mRNA expression of CD105 in (A) ccRCC, (B) pRCC and (C) chRCC tissues with different M stage (left panel), T stage (middle panel) and pathological stage (right panel). RCC, renal cell carcinoma; ccRCC, clear cell RCC; pRCC, papillary RCC; chRCC, chromophobe RCC; M, metastasis; T, tumor.

the two groups $(\mathrm{P}=0.003)$. These results demonstrated that ccRCC patients with a higher copy number of CD105 in their tumor tissues survive for longer. In chRCC, the same trend was identified, but it was not significant. However, in pRCC and chRCC, no significant impact of the CD105 copy number on survival was noted, but there was a trend, with those pRCC cases with a lower copy number of CD105 surviving for longer (Fig. 4).
Enriched pathways by genes associated with CD105 expression in RCC. Pathways enriched by genes positively and negatively correlated with CD105 expression in ccRCC were identified using webMeV. The 10 most enriched pathways by genes positively and negatively associated with the expression of CD105 were identified by analysis of the TCGA data for ccRCC (Fig. 5). The top 3 pathways are translation, eukaryotic translation initiation and cap-dependent translation initiation. 
Table II. Top 20 genes whose expression is associated with CD105.

\begin{tabular}{|c|c|c|c|c|c|c|}
\hline Gene name & $\log \mathrm{FC}$ & AveExpr & P-value & Adj. P-value & $\mathrm{t}$ & B \\
\hline UMPS & -2.4428 & 1.1268 & $3.22 \times 10^{-0.2}$ & 0.166 & -2.1469 & -3.8354 \\
\hline RAB25 & -2.1835 & 0.8057 & $1.00 \times 10^{-0.3}$ & 0.0223 & -3.3118 & -0.8953 \\
\hline SERPINA5 & -2.1075 & 2.8337 & $3.77 \times 10^{-0.2}$ & 0.1824 & -2.083 & -3.8258 \\
\hline KNG1 & -2.0606 & 0.8968 & $3.39 \times 10^{-0.2}$ & 0.1712 & -2.1264 & -3.866 \\
\hline ATP6V0A4 & -1.9947 & 0.5582 & $1.49 \times 10^{-0.2}$ & 0.1053 & -2.4422 & -3.2189 \\
\hline SSU72 & -1.9548 & 2.0074 & $3.37 \times 10^{-0.2}$ & 0.1706 & -2.1287 & -3.7714 \\
\hline CLDN8 & -1.8909 & 0.0309 & $1.99 \times 10^{-0.2}$ & 0.1249 & -2.3351 & -3.4854 \\
\hline FXYD4 & -1.8854 & 0.3206 & $3.24 \times 10^{-0.2}$ & 0.1666 & -2.1447 & -3.8588 \\
\hline SCNN1B & -1.7532 & 1.0789 & $8.90 \times 10^{-0.3}$ & 0.0761 & -2.6263 & -2.7293 \\
\hline ATP6V1G3 & -1.7428 & -0.287 & $1.83 \times 10^{-0.2}$ & 0.119 & -2.366 & -3.4324 \\
\hline SLC6A4 & 1.9869 & 4.3231 & $2.08 \times 10^{-0.2}$ & 0.1279 & 2.3183 & -3.6399 \\
\hline PTHLH & 1.8662 & 3.3825 & $5.00 \times 10^{-0.3}$ & 0.0541 & 2.8193 & -2.3873 \\
\hline DOC2A & 1.7541 & 3.6568 & $1.00 \times 10^{-0.4}$ & 0.0066 & 3.9825 & 1.316 \\
\hline MSLN & 1.7275 & 1.1849 & $1.04 \times 10^{-0.2}$ & 0.084 & 2.5701 & -2.8679 \\
\hline $\mathrm{ABCC} 2$ & 1.684 & 3.1759 & $1.00 \times 10^{-0.4}$ & 0.0075 & 3.8833 & 0.9659 \\
\hline ADCY2 & 1.6699 & 0.2704 & $1.00 \times 10^{-0.4}$ & 0.0057 & 4.0427 & 1.5383 \\
\hline PABPC1L & 1.6646 & 3.1852 & $0.00 \times 10^{-0}$ & 0.0033 & 4.3249 & 2.6437 \\
\hline RAB42 & 1.6581 & 4.0105 & $7.25 \times 10^{-6}$ & 0.0021 & 4.5307 & 3.4781 \\
\hline ATHL1 & 1.6556 & 5.3775 & $2.40 \times 10^{-3}$ & 0.035 & 3.0554 & -1.7662 \\
\hline GPR143 & 1.648 & 2.1146 & $3.00 \times 10^{-4}$ & 0.013 & 3.5988 & 0.012 \\
\hline
\end{tabular}

The data normalized with logarithmic scale. FC, fold change; Adj., adjusted; AveExpr, average expression.
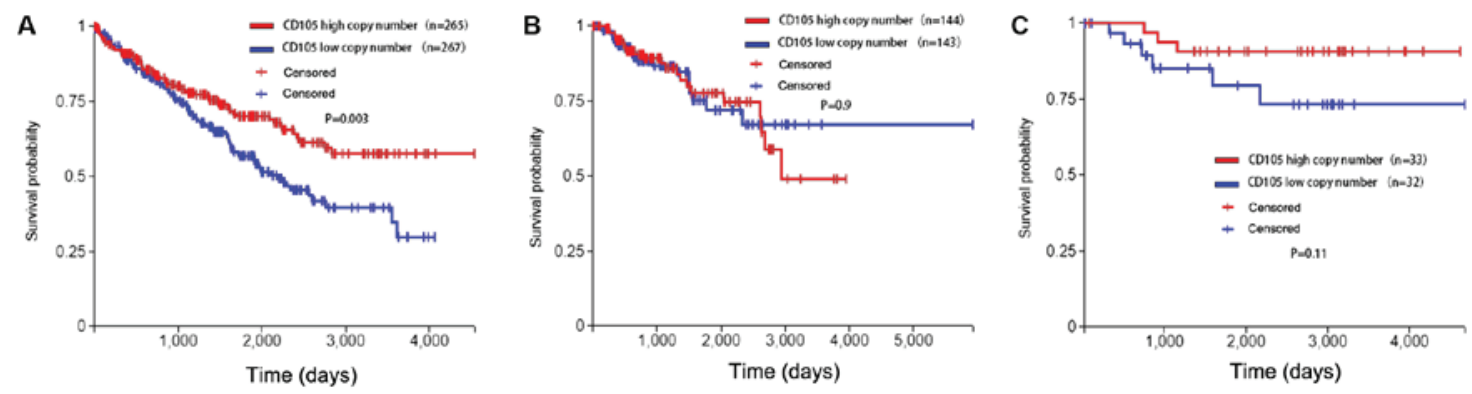

Figure 4. Copy number of CD105 and its association with survival in ccRCC, pRCC and chRCC. Kaplan-Meier analysis for the influence of the copy number of CD105 on overall survival in (A) ccRCC, (B) pRCC and (C) chRCC. RCC, renal cell carcinoma; ccRCC, clear cell RCC; pRCC, papillary RCC; chRCC, chromophobe RCC.

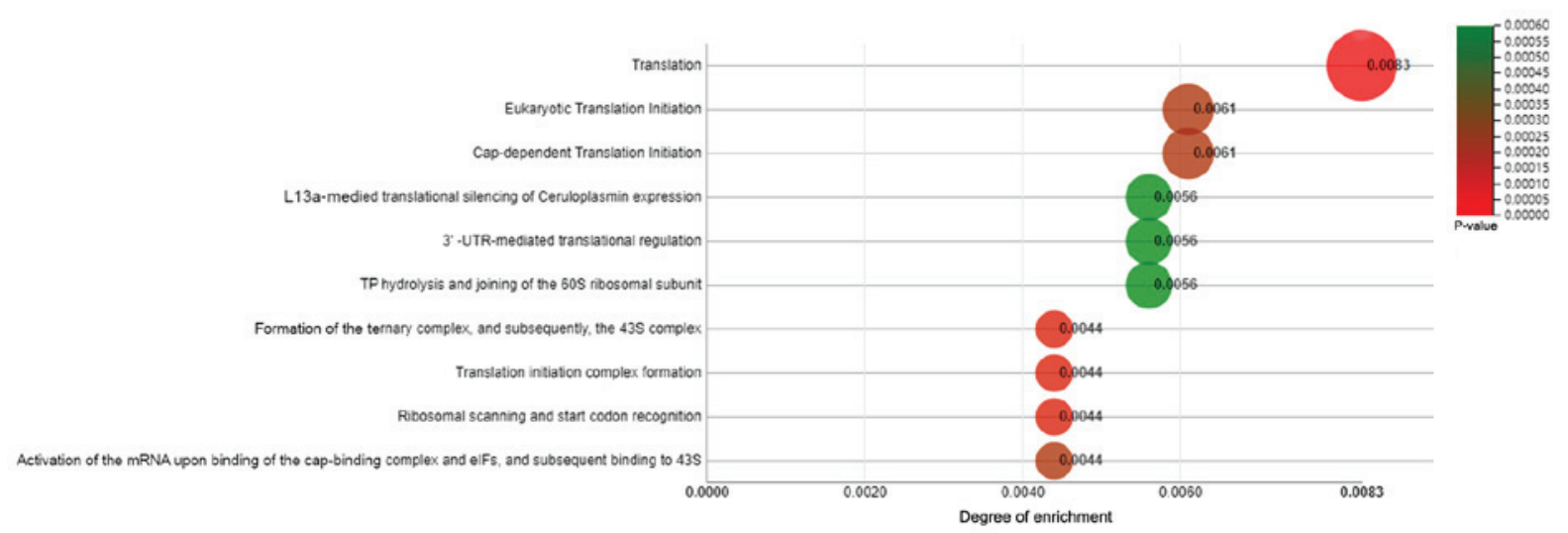

Figure 5. Top 10 pathways enriched by CD105-associated genes identified by webMeV in clear cell renal cell carcinoma. The size of the circle is proportional to the ratio. UTR, untranslated region. 
These results suggest that CD105 may be involved in translation pathway.

A total of 2,674 genes whose expression was correlated with CD105 expression in ccRCC were identified using webMeV. The top 20 of these genes are listed in Table II. Of note, CSC marker genes, including Nanog, sex-determining region Y box 2 and Oct4, were not among them.

\section{Discussion}

CD105 is a tumor marker expressed in vascular endothelial cells and has a role in new blood vessel formation (14); furthermore, CD105 is associated with high tumor microvessel density and is a predictor of poor prognosis in several solid tumor types (15). Recently, CD105 has been described as a renal CSC marker. Saeednejad Zanjani et al (13) reported that CD105 may serve as a useful prognostic molecular marker and potentially a target molecule for targeted therapy only in ccRCC, but possibly not in other subtypes of RCC. The present study identified that the expression of CD105 in tumor tissues is higher than that in normal tissues only in ccRCC, while, in pRCC and chRCC, the expression of CD105 is lower in tumor tissue compared with that in normal tissue. These results demonstrated that as a tumor marker, CD105 may only have a role ccRCC, but possibly not in other subtypes of RCC, which consistent with the results of the previous study (13). In the present study, even for ccRCC, the Kaplan-Meier survival curves of patients stratified by high and low expression of CD105 exhibited no significant difference. The reason for this observation may be the fact that the mRNA levels of CD105 may not represent the protein levels. Therefore, the CD105 copy number was used, based on which the ccRCC patients were stratified into two groups. It was revealed that the high CD105 copy number group survived for longer and this trend was in accordance with the in the high CD105 mRNA group. This appears in contrast with previous experimental results, where higher CD105 expression indicated more invasion and poor prognosis (20). Of note, previous studies also suggested that CD105 is an independent predictive marker for the risk of death and unfavourable prognosis in patients with ccRCC after curative resection $(21,22)$. The reason for this may be that hypoxia has an important role in ccRCC, and certain proteins are markedly decreased under hypoxia. In addition, CD105 may directly or indirectly regulate Hif-1 $\alpha$ under hypoxia, which may lead to more aggressive RCC phenotypes and a higher risk of recurrence (23). However, the precise mechanisms require further study.

In the present study, webMeV analysis was employed to identify pathways enriched by genes positively and negatively correlated with CD105 expression in ccRCC. The top 3 pathways identified were translation, eukaryotic translation initiation and cap-dependent translation initiation. As a CSC marker, CD105 confers self-renewal capacity and contributes to chemoresistance in RCC, and is associated with cell proliferation (24). Therefore, it is conceivable that CD105 is associated with translation, eukaryotic translation initiation and cap-dependent translation initiation. Furthermore, the genes most associated with CD105 were not cancer stem markers, which may indicate that CD105 exhibits stem cell-independent functions.
Several previous studies have indicated that anti-CD105 monoclonal antibody may effectively reduce or suppress angiogenesis, tumor growth and metastasis in SCID mice (25). In the present study, it was indicated that the role of CD105 in different stages of ccRCC is complex. In addition to the expression, the function of CD105 in RCC should also be evaluated to elucidate its role. CD105 may have a role in regulating the tumor microenvironment, such as hypoxia.

Of note, the present study had certain limitations. First, CD105 not only serves as a CSC marker in RCC, but also activates angiogenesis-associated factors in RCC. However, in the present study, the authors only assessed the expression in RCC tissue and its role in RCC. Furthermore, the present study did not include any in vitro cell experiment or animal study, and the expression of CD105 was only evaluated by a bioinformatics analysis to demonstrate the function of CD105 in RCC. Finally, hypoxia has an important role in $\mathrm{RCC}$, so it is possible that CD105 has different functions in different microenvironments, which requires further assessment.

In conclusion, the present results suggest that the roles of CD105 in RCC are complex. In ccRCC, CD105 mRNA expression was significantly upregulated and a higher copy number was significantly associated with a favourable prognosis. To evaluate the role of CD105 in RCC, it is not reasonable to only assess the expression and not the function. Of note, the association of CD105 mRNA expression and copy number with various types of RCC, their association with patient survival and the underlying mechanisms require further study.

\section{Acknowledgements}

The authors would like to thank Mrs. Karen Wolf (George Whipple Lab for Cancer Research, Departments of Urology and Pathology, University of Rochester, Rochester, NY, USA), a native English language speaker, for to checking the manuscript for grammatical and spelling errors.

\section{Funding}

The current study was funded by the National Natural Science Foundation of China (grant no. 81802518).

\section{Availability of data and materials}

The datasets generated and/or analyzed during the current study are available in The Cancer Genome Atlas repository (http://xena.ucsc.edu/).

\section{Authors' contributions}

DHS and JPC designed the study, collected the data and analyzed the data. DHS wrote the manuscript. YY and BP collected the data. CCG and XDY designed the study and collected the data.

\section{Ethics approval and informed consent}

Not applicable. 


\section{Patient consent for publication}

Not applicable.

\section{Competing interests}

The authors state that no competing financial interests exist.

\section{References}

1. Rini BI, Campbell SC and Escudier B: Renal cell carcinoma. Lancet 373: 1119-1132, 2009.

2. Siegel RL, Miller KD and Jemal A: Cancer statistics, 2017. CA Cancer J Clin 67: 7-30, 2017.

3. King SC, Pollack LA, Li J, King JB and Master VA: Continued increase in incidence of renal cell carcinoma, especially in young patients and high grade disease: United States 2001 to 2010. J Urol 191: 1665-1670, 2014.

4. Linehan WM and Ricketts CJ: Decade in review-kidney cancer: Discoveries, therapies and opportunities. Nat Rev Urol 11: 614-616, 2014.

5. Bedke J, Gauler T, Grünwald V, Hegele A, Herrmann E, Hinz S, Janssen J, Schmitz S, Schostak M, Tesch H, et al: Systemic therapy in metastatic renal cell carcinoma. World J Urol 35: 179-188, 2017.

6. Brown C: Targeted therapy: An elusive cancer target. Nature 537 (Suppl): S106-S108, 2016.

7. Yang F, Wu Q, Zhang Y, Xiong H, Li X, Li B, Xie W, Zhang L, Xu M, Zhang K and He F: LncRNA LOC653786 promotes growth of RCC cells via upregulating FOXM1. Oncotarget 9: 12101-12111, 2018

8. Choueiri TK and Motzer RJ: Systemic therapy for metastatic renal-cell carcinoma. N Engl J Med 376: 354-366, 2017.

9. Al-Hajj M and Clarke MF: Self-renewal and solid tumor stem cells. Oncogene 23: 7274-7282, 2004.

10. Corrò $\mathrm{C}$ and Moch $\mathrm{H}$ : Biomarker discovery for renal cancer stem cells. J Pathol Clin Res 4: 3-18, 2018.

11. Liu L, Wang Q, Mao J, Qin T, Sun Y, Yang J, Han Y, Li L and Li Q: Salinomycin suppresses cancer cell stemness and attenuates TGF- $\beta$-induced epithelial-mesenchymal transition of renal cell carcinoma cells. Chem Biol Interact 296: 145-153, 2018.

12. Matak D, Brodaczewska KK, Szczylik C, Koch I, Myszczyszyn A, Lipiec M, Lewicki S, Szymanski L, Zdanowski R and Czarnecka AM: Functional significance of CD105-positive cells in papillary renal cell carcinoma. BMC Cancer 17: 21, 2017.

13. Saeednejad Zanjani L, Madjd Z, Abolhasani M, Shariftabrizi A, Rasti A and Asgari M: Expression of CD105 cancer stem cell marker in three subtypes of renal cell carcinoma. Cancer Biomark 21: 821-837, 2018.
14. Duff SE, Li C, Garland JM and Kumar S: CD105 is important for angiogenesis: Evidence and potential applications. FASEB J 17: 984-992, 2003.

15. Dallas NA, Samuel S, Xia L, Fan F, Gray MJ, Lim SJ and Ellis LM: Endoglin (CD105): A marker of tumor vasculature and potential target for therapy. Clin Cancer Res 14: 1931-1937, 2008.

16. Bussolati B, Bruno S, Grange C, Ferrando U and Camussi G: Identification of a tumor-initiating stem cell population in human renal carcinomas. FASEB J 22: 3696-3705, 2008.

17. Hasmim M, Bruno S, Azzi S, Gallerne C, Michel JG, Chiabotto G, Lecoz V, Romei C, Spaggiari GM, Pezzolo A, et al: Isolation and characterization of renal cancer stem cells from patient-derived xenografts. Oncotarget 7: 15507-15524, 2016.

18. Song L, Ye W, Cui Y, Lu J, Zhang Y, Ding N, Hu W, Pei H, Yue Z and Zhou G: Ecto-5'-nucleotidase (CD73) is a biomarker for clear cell renal carcinoma stem-like cells. Oncotarget 8: 31977-31992, 2017.

19. Feldman DR and Motzer RJ: Novel targets and therapies for metastatic renal cell carcinoma. Oncology (Williston Park) 20: 1745-1756, 2006

20. Yang X, Zhang D, Chong T, Li Y, Wang Z and Zhang P: Expression of CK19, CD105 and CD146 are associated with early metastasis in patients with renal cell carcinoma. Oncol Lett 15: 4229-4234, 2018.

21. Yagasaki H, Kawata N, Takimoto $\mathrm{Y}$ and Nemoto $\mathrm{N}$ : Histopathological analysis of angiogenic factors in renal cell carcinoma. Int J Urol 10: 220-227, 2003.

22. Saroufim A, Messai Y, Hasmim M, Rioux N, Iacovelli R, Verhoest G, Bensalah K, Patard JJ, Albiges L, Azzarone B, et al: Tumoral CD105 is a novel independent prognostic marker for prognosis in clear-cell renal cell carcinoma. Br J Cancer 110: 1778-1784, 2014.

23. Myszczyszyn A, Czarnecka AM, Matak D, Szymanski L, Lian F, Kornakiewicz A, Bartnik E, Kukwa W, Kieda C and Szczylik C: The role of hypoxia and cancer stem cells in renal cell carcinoma pathogenesis. Stem Cell Rev 11: 919-943, 2015.

24. Hu J, Guan W, Liu P, Dai J, Tang K, Xiao H, Qian Y, Sharrow AC, $\mathrm{Ye} \mathrm{Z}, \mathrm{Wu} \mathrm{L}$ and $\mathrm{Xu} \mathrm{H}$ : Endoglin is essential for the maintenance of self-renewal and chemoresistance in renal cancer stem cells. Stem Cell Reports 9: 464-477, 2017.

25. Seon BK, Matsuno F, Haruta Y, Kondo M and Barcos M: Long-lasting complete inhibition of human solid tumors in SCID mice by targeting endothelial cells of tumor vasculature with antihuman endoglin immunotoxin. Clin Cancer Res 3: 1031-1044, 1997.

This work is licensed under a Creative Commons Attribution-NonCommercial-NoDerivatives 4.0 International (CC BY-NC-ND 4.0) License. 